\title{
Predicting Coordinated Group Movements of Sharks with Limited Observations using AUVs
}

\author{
Cherie Ho \\ Dept. of Engineering \\ Harvey Mudd College \\ Claremont, CA 91711 \\ chho@g.hmc.edu
}

\author{
Kimberly Joly \\ Dept. of Engineering \\ Harvey Mudd College \\ Claremont, CA 91711 \\ kjoly@g.hmc.edu
}

\author{
Andrew P. Nosal \\ Natural Sciences Dept. \\ University of Saint Katherine \\ San Marcos, CA 92069 \\ anosal@skcca.edu
}

\author{
Christopher G. Lowe \\ Dept. of Biological Sciences \\ CSU Long Beach \\ Long Beach, CA 90840 \\ clowe@csulb.edu
}

\author{
Christopher M. Clark \\ Dept. of Engineering \\ Harvey Mudd College \\ Claremont, CA 91711 \\ clark@hmc.edu
}

\begin{abstract}
This paper presents a method for modeling and then tracking the $2 \mathrm{D}$ planar size, location, orientation, and number of individuals of an animal aggregation using Autonomous Underwater Vehicles (AUVs). It is assumed that the AUVs are equipped with sensors that can measure the position states of a subset of individuals from within the aggregation being tracked. A new aggregation model based on provably stable Markov Process Matrices is shown as a viable model for representing aggregations. Then, a multi-stage state estimation architecture based on Particle Filters is presented that can estimate the time-varying model parameters in real-time using sensor measurements obtained by AUVs. To validate the approach, a historical data set is used consisting of $>100$ shark trajectories from a leopard shark aggregation observed in the La Jolla, CA coast area. The method is generalizable to any stable group movement model constructed using a Markov Matrix. Simulation results show that, when at least $40 \%$ of sharks are tagged, the estimated number of sharks in the aggregation has an error of $6 \%$. This error increased to $27 \%$ when the system was tested with real data.
\end{abstract}

\section{CCS Concepts}

-Computing methodologies $\rightarrow$ Multi-agent systems; Intelligent agents; Cooperation and coordination; Mobile agents;

\section{Keywords}

Multi-Target Tracking, Aggregation Modeling, Swarms

\section{INTRODUCTION}

Permission to make digital or hard copies of all or part of this work for personal or classroom use is granted without fee provided that copies are not made or distributed for profit or commercial advantage and that copies bear this notice and the full citation on the first page. Copyrights for components of this work owned by others than the author(s) must be honored. Abstracting with credit is permitted. To copy otherwise, or republish, to post on servers or to redistribute to lists, requires prior specific permission and/or a fee. Request permissions from permissions@acm.org.

SAC 2017, April 03 - 07, 2017, Marrakech, Morocco

(C) 2017 Copyright held by the owner/author(s). Publication rights licensed to ACM. ISBN 978-1-4503-4486-9/17/04 . .\$15.00

DOI: http://dx.doi.org/10.1145/3019612.3019711
Studying the spatial interactions of individuals within an animal aggregation provides insight to the group level dynamics. Work done by various research teams has recently shown that multiple AUVs can cooperatively and autonomously track and follow a marine target tagged with an acoustic transmitter $[1,2]$. However, these works focus on fine-scale localization and characterization of the movements of only the individuals within a group that are tagged (i.e. those that can be observed). In contrast, the goal of many marine biologists is to determine the movement behaviors and the nature of association for a population of aggregating individuals while in a particular habitat (i.e. Fig. 1). Understanding these motion behaviors, including the movement pattern of the entire aggregation as well as the individuals that form the aggregation, is essential for monitoring and protecting marine habitats and associated resources.

This work aims to provide a new tool for tracking whole aggregations of animals, despite only having access to observations of a subset of individuals within the aggregation. Specifically, the contributions of this work include:

- A new animal aggregation model based on provably stable Markov Process Matrices, that can be constructed from real animal position data or artificial potential field simulations.

- A multi-stage state estimation architecture based on Particle Filters that estimates aggregation model parameters including size, position, orientation, and number of individuals in the aggregation using sensor measurements obtained by AUVs.

- Validation of the model and filter using an aerial video data set of a leopard shark aggregation.

\section{BACKGROUND}

Many marine species exhibit aggregation behavior that form from either social interactions and/or result from constraining or preferred environmental conditions. This behavior results in group-level motion behaviors. For example, a highly polarized shoal results in schooling behavior that protects individuals within the group from predators as well as enhancing foraging abilities [3]. Understanding individual movements within an aggregation can lead to deeper insights into the social behavior of the species and how individuals respond to changing environmental conditions. 
Movement patterns of a wide variety of marine animals have been extensively studied to produce mathematical models that describe an aggregation's group dynamic $[4,5$, $6,7,8,9]$. Theoretical work has been done to model the collective behavior within a fish school. Many of such models are based on the physics-inspired model where individuals of an aggregation interact through attraction, repulsion and alignment $[4,5,6,7]$. The movement patterns of leopard sharks in response to changing water temperature has been extensively studied [10,11]. Observations have shown that sharks prefer the warmest areas of an environment and move to warmer locations over the course of the day [10]. Tracking fish aggregations has been done more often in captivity and on individuals that are small in size $[12,13,14]$. Most often, image processing techniques coupled with filtering algorithms have been used to automatically detect and track fish schools from video data. For example, in [15], image processing is first used to identify possible fish heads, then filtering and feature matching are used to track the fish. Finally the feature information is processed through trajectory linking. To better approximate the uncertainty of fish motions and improve position estimate, Particle Filters (PF) were introduced to use a hybrid motion model [16].

Within the context of tracking individual large marine animals in natural habitats, conventional methods include tagging the individuals with acoustic transmitters (tags). Unlike small species that can be easily tracked and their movements characterized in a lab, larger and more mobile species require acoustic tracking to quantify their movements. The acoustic transmissions from these tags are detected by an array of hydrophones, and the difference in time-of-arrival of the transmission between hydrophones in the array is used to estimate the position of the individual [17, 18]. Alternatively, active tracking of a marine target can be done by mounting hydrophones on a boat and following it with a boat to get position estimates [19]. With the advancement of technology, tracking a marine animal can be automated by using AUVs [20, 21, 22]. A multiple AUV system can be used to track and follow a marine target autonomously [1, 2]. However, the work has been limited to tracking a single marine target, not entire aggregations. In addition, a single AUV has demonstrated the capability of tracking six sharks simultaneously for a continuous 1-3 hours [23]. However, the AUV was not able to estimate the relative positions of other sharks within the aggregation.

Unlike the works cited above, this research uses a transition matrix generated from a stochastic movement model to indicate the likelihood an individual will be a certain distance from the aggregation, and leverages the resulting probability density function to estimate the number of sharks in an aggregation. The work also generalizes to any movement model that can generate a stable transition matrix.

\section{MOTIVATING DATA SET}

A historical data $H$ was acquired by applying an image processing algorithm to video footage of aggregations of sharks filmed by a GoPro attached to a $30 \mathrm{~m}$ high helium balloon. The video frames include approximately 200 sharks in a 20 by 30 meter surface area. The video consists of two 17 minute video clips and was acquired on June $5^{t h} 2014$.

The image processing algorithm employs two steps to extract shark trajectories from the video image frames. The first step applies a threshold filter to produce sharp con-

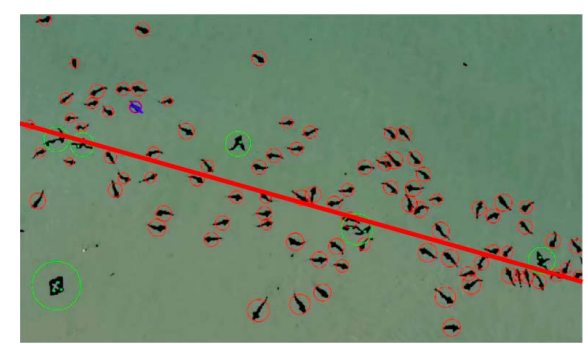

(a)

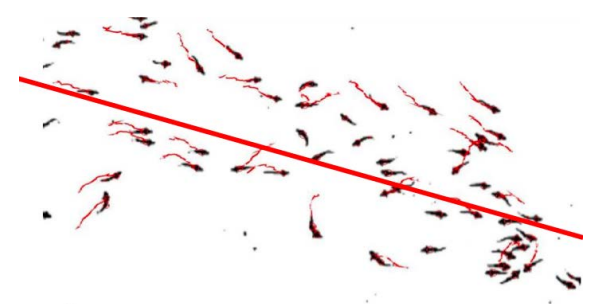

(b)

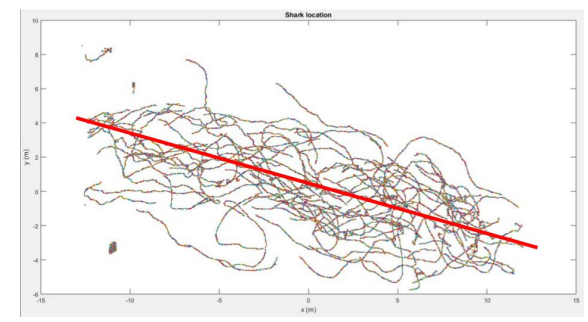

(c)

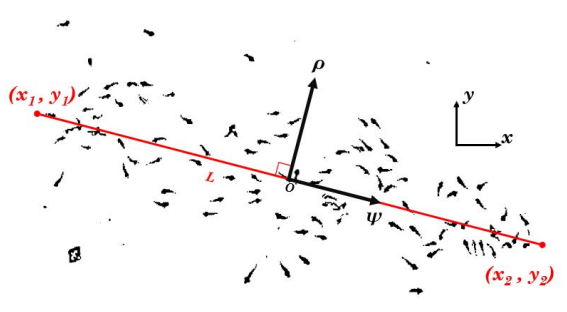

(d)

Figure 1: Cropped aerial image of a leopard shark aggregation with 112 sharks(a). A threshold filter is applied to the video and the position of each shark is indicated by a red line, (b). In (c), the $\mathrm{x}$ and $\mathrm{y}$ coordinates of each shark are plotted. The attraction line is indicated by the red line. Aggregation model parameters are shown in $(d)$.

trast images with (black) sharks on a (white) background, as shown in Fig. 1(b). Standard blob tracking is applied to the threshold images to construct shark trajectories, by using the Matlab Computer Vision System Toolbox.

The algorithm's second step uses a Kalman Filter (KF) for tracking each individual shark in the aggregation. In this case, the KF outputs the $2 \mathrm{D}$ position, orientation, and velocity of each shark. The KF follows a standard prediction step/correction step structure. Specifically, for each video image frame, shark states are predicted using previous velocity and orientation estimates. Shark position states are corrected using blob centroid positions, and shark orienta- 
tions are corrected using the blob major axis orientations. The algorithm also uses the blob size, which corresponds to the number of pixels covered by a shark, to determine if the blob includes observations of multiple sharks. An example output of this step is illustrated by red lines in Fig. 1(c).

Lastly, a polynomial regression calibration algorithm is applied to the shark trajectories to convert a shark's location and velocity from pixel to meters, and correct the space distortion from the fish eye camera lens.

\section{PROBLEM DEFINITION}

The goal of this project is to estimate the $2 \mathrm{D}$ planar size, location, orientation, and number of individuals of an animal aggregation using robots equipped with sensors that can measure the position states of a subset of individuals within the aggregation. For this particular problem, as shown in Fig. 1(d), we represent the aggregation with a line of attraction (red line in Fig. 1) with position end points $x_{1}, y_{1}, x_{2}$, $y_{2}$ where there is a favorable temperature zone [11], length $L$, and number of animals $n$. The state of the attraction line end points at time $t$ is denoted by $X_{t}^{\text {line }}$. The state of the aggregation number of individuals and line length at time $t$ is denoted by $X_{t}^{a g g}$. The paper introduces a pair of coordinate axes based on the attraction line, with $\rho$ as the distance from the line and $\psi$ as the distance along the line from the line's center.

Given: A set $H$ of historical data sets, each consisting of xy position data of individuals within aggregations, as well real-time position measurements $Z_{t}$ of $m$ tagged individuals within the aggregation of $n$ individuals being tracked:

$$
Z_{t}=\left\{Z_{i, t} \mid i \in(1, m), t \in(1, T)\right\}
$$

Determine: The time-varying attraction line and aggregation states:

$$
\begin{aligned}
X_{\text {line }, t} & =\left\{\left[x_{1}, y_{1}, x_{2}, y_{2}\right]_{t} \mid t \in(1, T)\right\} \\
X_{\text {agg }, t} & =\left\{[n, L]_{t} \mid t \in(1, T)\right\}
\end{aligned}
$$

\section{PROPOSED METHOD}

The proposed system is summarized in the block diagram shown in Fig. 2. The historical data set $H$ is first processed to determine the parameters of a stable multi-agent swarm model. In this paper, we use a swarm model based on Artificial Potential Fields (APFs), where the model parameters are attractive and repulsive gains $\left(K_{a t t}, K_{r e p}, K_{h a b}\right)$.

The swarm model parameters are used in Transition Matrix Modeling to run a large number of simulations in which aggregation parameters (e.g. $n, L)$ are varied. From these simulations, Markov Matrix model parameters $\rho_{90}$ and $\psi_{90}$ are determined, which correspond respectively to the distance from the line, and the distance from the center along the line within which $90 \%$ of individuals in the aggregation are found. These parameters are essential for the Particle Filter (PF) correction step of the aggregation state estimation. Details are provided in subsequent sections.

During real time aggregation tracking with an AUV, new measurements $Z_{i, t}$ of $i=1 . . m$ tagged individuals are used by Individual Particle Filters to estimate the state of each tagged individual $X_{i, t}$ at time $t$. The estimated position states of the tagged individuals $X_{i, t}$ are used to estimate the attraction line state $X_{\text {line, } t}$ using the Attraction Line Particle Filter, where $X_{\text {line }, t}=\left[x_{1}, y_{1}, x_{2}, y_{2}\right]_{t}$.

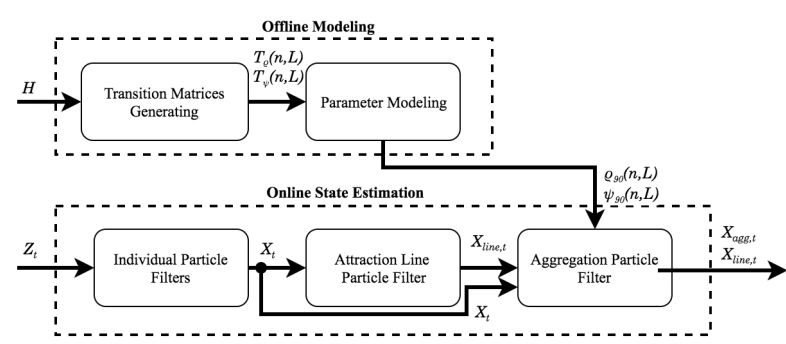

Figure 2: Overall system block diagram.

The estimated position states of the tagged individuals $X_{i, t}$ and the estimated line state $X_{\text {line, } t}$ are used to estimate the aggregation state $X_{a g g, t}$ at time $t$ using the $A g$ gregation Particle Filter. In this case, the aggregation state is defined by the swarm model's line segment of attraction, where $X_{a g g, t}=[n, L]_{t}$. In particular, states $X_{i, t}$ are used in the aggregation particle filter to update the measured values of $\rho_{90}$ and $\psi_{90}$ for comparison with each aggregation particle's expected $\rho_{90}$ and $\psi_{90}$.

\section{OFFLINE MODELING}

The goal of the offline modeling is to generate discrete time transition matrices, or Markov matrices, that model an individual's movement with respect a line of attraction. Specifically, the model includes two types of transition matrices, one that represents the likelihood an individual will increase or decrease its $\rho$ value, and a second transition matrix that represents the likelihood an individual will increase or decrease its $\psi$ value. Once the transition matrices are known, and they are shown to be stable, their steady state values can be used within the online state estimation described later to estimate aggregation parameters $n$ and $L$.

In the model proposed here, movements of each shark are modeled as transitions between the discretized values of $\rho$ and $\psi$. Fig. 3 illustrates the line of attraction (red line) and the discretized $\rho$ and $\psi$ values in (a) and (b) respectively. For example, In Fig. 3(a), the transition from $\rho_{2}$ to $\rho_{1}$ is indicated by the lowest curved arrow, and the likelihood of the shark making this transition is $T_{\rho}(2,1)$.

We define $p_{\rho, t}=\left[\begin{array}{lllllll}p_{\rho,-s} & \ldots & p_{\rho,-1} & p_{\rho, 1} & p_{\rho, 2} & \ldots & p_{\rho, s}\end{array}\right]_{t}$ to be a $s \times 1$ vector in which $p_{\rho_{i}}$ is the likelihood at time $t$ that the shark is at discretized distance $\rho_{i}$ with respect to the line of attraction. The linear transition matrix $T_{\rho}$ encodes the likelihood of transitions between discrete $\rho$ values. That is the $i, j^{t h}$ element of $T_{\rho}$ is the likelihood of a shark transitioning from $\rho_{i}$ to $\rho_{j}$ at time step $t$.

$$
\begin{aligned}
p_{\rho, t} & =T_{\rho} p_{\rho, t-1} \\
& =\left(T_{\rho}\right)^{t} p_{\rho, 0}
\end{aligned}
$$

Vector $p_{\psi, t}$ and matrix $T_{\psi}$ can similarly be defined. Notably, the transition Matrices $T_{\rho}$ and $T_{\psi}$ are dependent on the number of sharks $n$ in the aggregation and the line of attraction length $L$.

If the system described by Equation 1 is stable, then $p_{\rho, t}$ converges to a steady state probability vector $p_{\rho, \infty}$.

$$
p_{\rho, \infty}=T_{\rho} p_{\rho, \infty}
$$

For the system to be stable, the transition matrices obtained must be Markov Matrices which have a maximum absolute 
eigenvalue of 1. By the Perron-Frobenius theorem [24], the eigenvector $p_{\rho, \infty}$ corresponding to the eigenvalue of 1 is the stable equilibrium distribution of the Markov matrix. As the eigenvalue of 1 will be dominant, the probability distribution will converge to the steady state probability $p_{\rho, \infty}$ regardless of the initial distribution.

\subsection{Transition Model Generating}

Two methods are presented for generating transition models. One method generates the transition matrices $T_{\rho}$ and $T_{\psi}$ directly from historical data set shark trajectories. To be specific, each shark trajectory of the motivating data set is used. For each time step of each trajectory, the shark's transition from its current discretized $\rho_{i, t}$ to its next $\rho_{j, t+1}$ is recorded. The transition likelihood for each entry in the transition matrix is then calculated as in Eq. 3, where $\delta_{s, i, j, t}$ equals 1 if shark $s$ transitioned from $\rho_{i}$ to $\rho_{j}$ at time $t$, and equals 0 otherwise.

$$
T_{\rho}(i, j)=\frac{\sum_{s} \sum_{t} \delta_{s, i, j, t}}{\sum_{s} \sum_{k} \sum_{t} \delta_{s, i, k, t}}
$$

A second method for generating transition matrices can be used when a historical data set is not available, but a well known swarm model for the animals of interest exists. In this later case, the transition matrices can be generated from the shark trajectories outputted from swarm model simulations. In this paper, we use an Artifical Potential Field (APF) [4] swarm model to produce shark trajectories.

In the APF model, simulated sharks move in response to forces created by both attractive artificial potentials as well as repulsive potentials. In this case sharks are attracted to both the line of attraction as well as other sharks. Once the sharks are too close to each other, they feel a repulsive force from each other. This model is shown in Fig. 3(c), which illustrates the behavioral zones at which attraction and repulsion occurs [4]. In the model, each shark updates its direction vector $\vec{d}_{i, t}$ based on Equation 4 , where $\vec{r}_{i, j}$ represents the vector from individual $j$ to individual $i$.

$$
\vec{d}_{i, t}=\sum_{j=1}^{n} f_{i j} \frac{\vec{r}_{i, j}^{t-1}}{\left\|\vec{r}_{i, j}^{t-1}\right\|}+K_{h a b} \frac{\vec{r}_{i, \text { line }}^{t-1}}{\left\|\vec{r}_{i, \text { line }}^{t-1}\right\|}
$$

where

$$
f_{i j}= \begin{cases}-K_{r e p} & 0 \leq\left\|\vec{r}_{i, j}^{t-1}\right\| \leq r_{r e p} \\ 0 & r_{r e p} \leq\left\|\vec{r}_{i, j}^{t-1}\right\| \leq r_{n e u} \\ K_{a t t} & r_{n e u} \leq\left\|\vec{r}_{i, j}^{t-1}\right\| \leq r_{a t t} \\ 0 & r_{a t t} \leq\left\|\vec{r}_{i, j}^{t-1}\right\|<\infty\end{cases}
$$

Eq. 4 is used to update the position of sharks at each time step $t$ of a simulation. After a simulation has run for $100,000+$ iterations, the shark trajectories created during the simulation can be processed with Eq. 3 to generate transition matrices $T_{\rho}$ and $T_{\psi}$.

Regardless of the method used to generate transition matrices, there are two criteria that $T_{\rho}$ and $T_{\psi}$ must satisfy. First, the transition matrices must be stable in that their maximum eigenvalue is 1 . Second, the eigenvector $p_{\rho, \infty}$ associated with the eigenvalue of 1 represents the likelihood of a real shark in the aggregation being found at distances $\rho$ and $\psi$.

To validate that our transition matrix modeling from the motivating data set meets both criteria, we first observe that

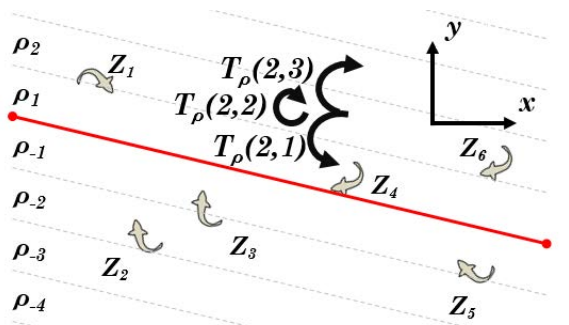

(a)

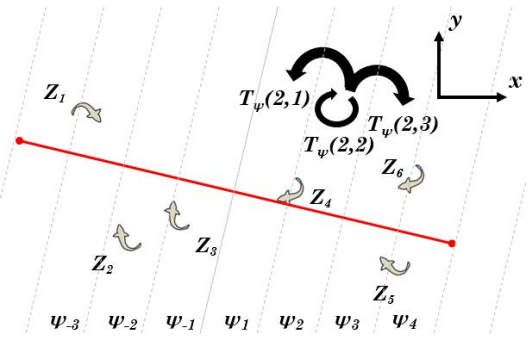

(b)

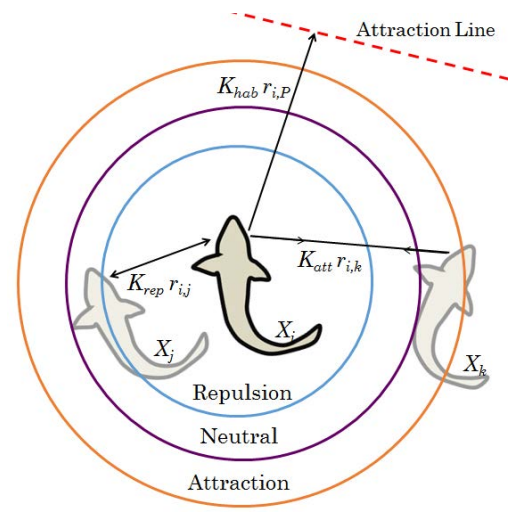

(c)

Figure 3: The shark movement space is discretized into bins of distances from the attraction line, as shown in (a). The shark movement space is discretized into bins of distances from the center along the line, as shown in (b). The two figures show the three possible transitions a shark originally at state 2 can make. In (c), A graphical representation of the artificial potential field model.

the maximum eigenvalues of both $T_{\rho}$ and $T_{\psi}$ are 1.0 and 1.0 respectively. In both cases the maximum eigenvalues are 1.0, which is expected given they are Markov matrices. Second, running lengthy (i.e. 100,000 time steps) simulations by randomly initializing shark positions and using Eq. 1 can generate a histogram of shark $\rho$ and $\psi$ values that match the original distribution as shown in Fig. 4.

\subsection{Parameter Modeling}

The transition matrices generated, and the associated steady state (eigen)vectors $p_{\rho, \infty}$ and $p_{\psi, \infty}$ will be used during the the on-line state estimation of aggregation parameters. Notably, the transition matrices and eigenvectors are functions of both the number of sharks $n$ as well as the length of the line of attraction $L$. For example, the more sharks in the aggregation, the greater the maximum value 


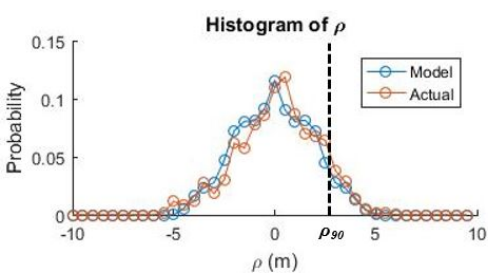

(a)

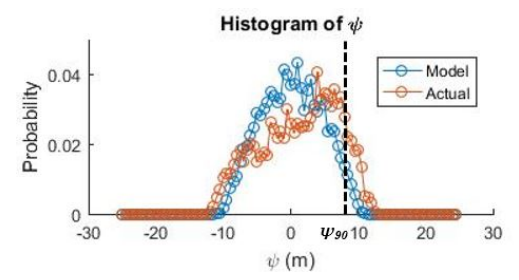

(b)

Figure 4: The histograms from model and actual are compared, in (a), for $\rho$ and, in (b), for $\psi$.

of $\rho$ that has non-negligible likelihood of containing sharks in the aggregation. With this in mind, we define $\rho_{90}(n, L)$ and $\psi_{90}(n, L)$ to be values of $\rho$ and $\psi$ that are expected to bound $90 \%$ of the steady state distributions $p_{\rho, \infty}$ and $p_{\psi, \infty}$. Fig. 4 illustrates these values for the motivating data set.

Given the shark trajectories from a large historical data set or swarm model simulations, one can calculate the transition matrices, associated eigenvectors, and hence $\rho_{90}$ and $\psi_{90}$ for different values of $n$ and $L$. Fig. 5(a) and Fig. 5(b) show the models of $\rho_{90}(n, L)$ and $\psi_{90}(n, L)$ as determined by Transition Matrix Modeling. Hence, for a given $n$ and $L$, there is an expected $\rho_{90}$ and $\psi_{90}$. During on-line state estimation, this will be used in the Particle Filter to estimate candidate values of $n$ and $L$ by comparing their expected values of $\rho_{90}$ and $\psi_{90}$ with those measured in real time.

\section{ONLINE STATE ESTIMATION}

The hierarchical state estimator is based on Particle Filtering, which estimates the aggregation state in three stages as shown in Fig. 2. In the first stage, sensor measurements of the tagged individuals from the AUVs are used to estimate shark positions $X_{t}$ with Individual Particle Filters. In the second stage, the position estimates $X_{i, t}$ of each tagged individual $i$ are used to estimate the attraction line orientation and position $X_{\text {line,t }}$ by the Attraction Line Particle Filter. In the final stage, the position estimates $X_{i, t}$ and estimated attraction line $X_{\text {line,t }}$ are used by the Aggregation Particle Filter to estimate the aggregation state $X_{a g g, t}$.

\subsection{Individual Particle Filters}

The state of each tagged individual is estimated with an Individual State Estimator that fuses measurements from sensors mounted on the AUVs. This leverages work done by previous researchers where a distributed PF was used to fuse measurements from AUV mounted hydrophones to estimate shark position[2]. Position estimates errors from their trials were documented to be on the order of $5 \mathrm{~m}$. Our work assumes AUVs will be available that have similar sensors.

\subsection{Attraction Line Particle Filter}

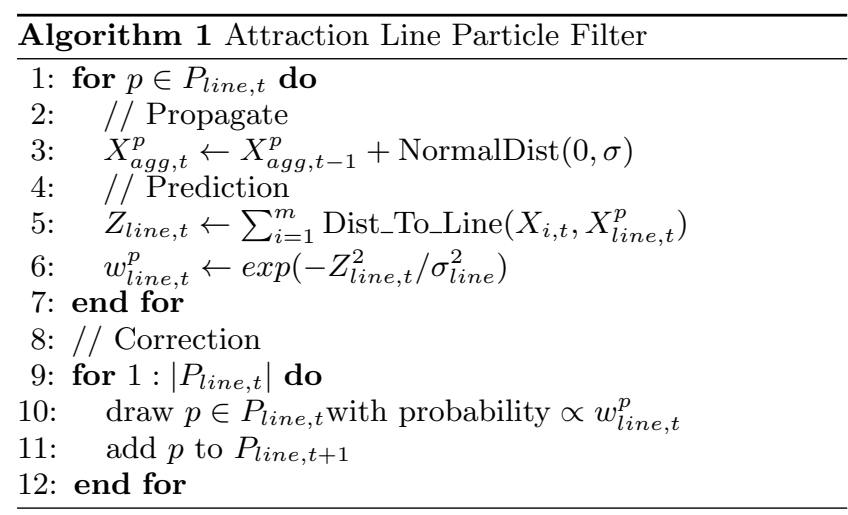

The Attraction Line PF represents the belief state of the aggregation at time $t$ with a set of particles denoted by $P_{\text {line }, t}$. Each particle $p \in P_{\text {line }, t}$ is represented by the set $X_{\text {line }, t}^{p}, w_{\text {line }, t}^{p}$, where $X_{\text {line }, t}^{p}$ is the particle's estimate of the attraction line state and $w_{\text {line, },}^{p}$ is its associated weight.

The attraction line state estimator uses the estimated shark positions in $X_{i, t}$ to calculate the line state $X_{l i n e, t}$. Each $p \in P_{\text {line }, t}$ is initialized with xy coordinates of line end points sampled randomly from uniform distributions. The algorithm for the attraction line particle filter is shown in Alg. 1. The relevant weight function and propagation parameters are found in Table 1.

At each time step $t$, the Particle Filter measures the total line error (Alg. 1, Lines 4-7). The attraction line particle weights $w_{\text {line,t }}^{p}$ are calculated as the conditional probability of obtaining shark states $X_{i, t}$, assuming the particle's line of attraction end points are correct. This conditional probability is a Gaussian function with measurement mean 0 and standard deviation $\sigma_{\text {line }}$, shown in Table 1.

\subsection{Aggregation Particle Filter}

The Aggregation Particle Filter represents the belief state of the aggregation at time $t$ with a set of particles denoted by $P_{a g g, t}$. Each particle $p \in P_{a g g, t}$ is represented by the set $X_{a g g, t}^{p}, w_{a g g, t}^{p}$, where $X_{a g g, t}^{p}$ is the particle's estimate of the aggregation state at time $t$ and $w_{a g g, t}^{p}$ is its associated weight. $X_{a g g, t}^{p}$ contains $n_{t}^{p}$ and $L_{t}^{p}$, which represents the particle's estimate of number of sharks and attraction line length respectively.

The aggregation state estimator takes in position estimates of the tagged sharks $X_{i, t}$ to estimate the aggregation state $X_{a g g, t}$. Each $p \in P_{a g g, t}$ is initialized with a number of sharks and an attraction line length that are sampled randomly from uniform distributions. The algorithm for the aggregation state estimator is shown in Alg. 2. The relevant weight function and propagation parameters are found in Table 1.

Alg. 2 shows that at each time step $t$, the state estimator iteratively updates the particle set $P_{a g g, t}$. In one time step, say $t$, the set of particles in $P_{a g g, t}$ are propagated randomly with a normal distribution (Alg. 2 Line 4-5).

Each particle's weight $w_{a g g, t}^{p}$ is determined using a moving window (of size $\tau$ ) of accumulated tagged shark measurements taken from time $t-\tau$ to time $t$. Using this accumulated data, histograms of measured $\rho$ and $\psi$ values are constructed from which the measured values of $\rho_{90}$ and $\psi_{90}$ are extracted as $Z_{\rho, t}$ and $Z_{\psi, t}$. These are compared with the expected values of $\rho_{90}\left(n_{t}^{p}, L_{t}^{p}\right)$ and $\psi_{90}\left(n_{t}^{p}, L_{t}^{p}\right)$ for each par- 


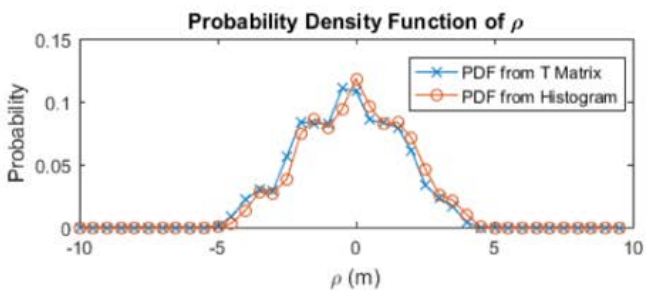

(a)

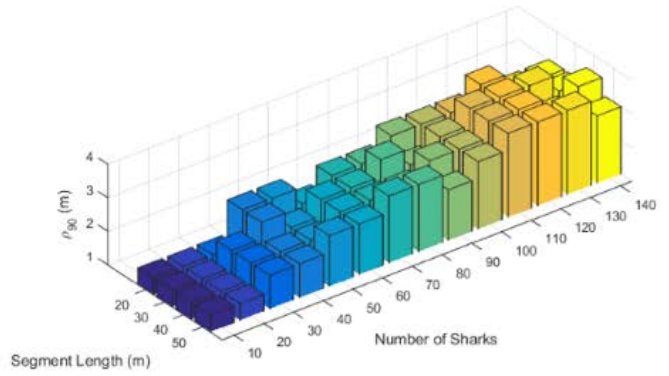

(c)

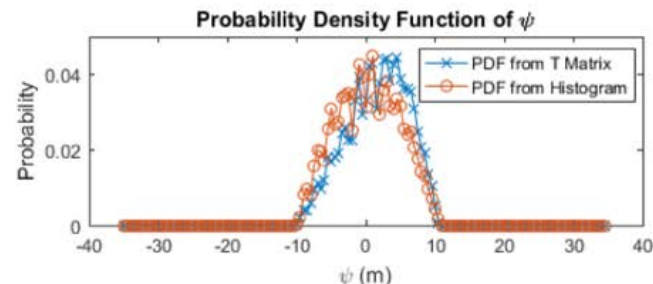

(b)

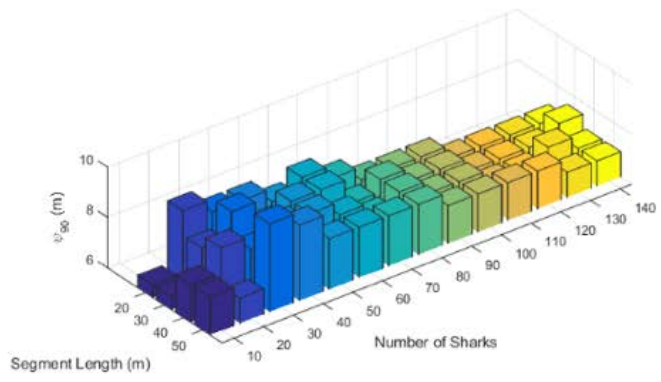

(d)

Figure 5: Comparison of $p_{\infty}$ and Histogram of (a) sharks' distance away from line (b) sharks' distance along the line from the center for $n=100$ and $L=40$. (c) $\rho_{90}(n, L)$ and (d) $\psi_{90}(n, L)$ from Transition Matrix Modeling.

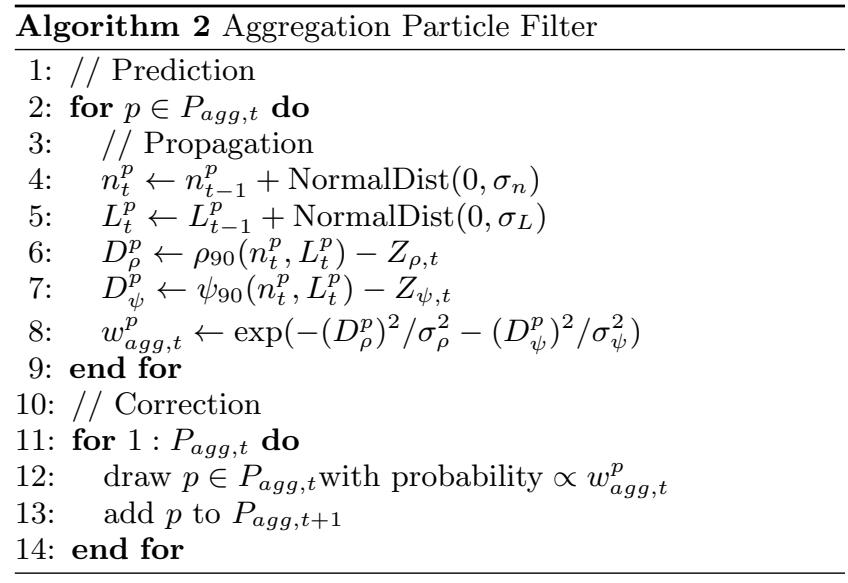

ticle, which are obtained from the transition models. The difference between the measured and expected values $D_{\rho, t}$ and $D_{\psi, t}$ are passed through a Gaussian function to obtain the particle weight. New particles $w_{a g g, t+1}^{p}$ are resampled using the particle weights $w_{a g g, t}^{p}$.

\section{RESULTS AND DISCUSSION}

The state estimation process is validated using both simulated and actual aggregation shark trajectories. The simulated aggregation trajectories were generated using the APF model. The actual aggregation trajectories were extracted from the motivating video data set of $n=112$ sharks with a $L=30 \mathrm{~m}$ attraction line described in Section 3. For both cases, the moving window parameter $\tau$ is the length of the data set. The aggregation state estimation is not real-time in either case, but the later case demonstrates how the the system will perform with actual shark aggregation data. The state estimation ran on a Windows computer with a Intel Core i5-3570K processor @ 3.40 GHz and 7.89GB RAM. The state estimation (50 particles for attraction line PF and aggregation $\mathrm{PF}$ ) took 0.455 seconds per algorithm iteration, each of which processed observations of 100 tagged sharks.

\subsection{Simulation Data Results}

Simulations of aggregations with 100 sharks $(n=100)$ and a line of attraction length $L=40 \mathrm{~m}$ were conducted. During simulations, the sharks followed the APF model using the gain values shown in Table 1 . Results from the simulation are shown in Fig. 6 and summarized in Table 1. To note, the line error at time $t$ is calculated by:

$$
\epsilon_{t}^{\text {line }}=\frac{\sum_{n=1}^{\infty} \sqrt{\left\|\vec{r}_{i, \text { line }_{e s t}}\right\|-\left\|\vec{r}_{i, \text { line }_{a c t}}\right\|^{2}}}{n}
$$

The RMS state estimation errors are found in Table 2. As shown in Fig. 6, when at least $40 \%$ of sharks are tagged, the attraction line, the number of sharks and attraction line length are estimated well. To be specific, the line position estimation errors are consistently less than the threshold of $0.1 \mathrm{~m} /$ shark. The root mean squared (RMS) error of estimated number of sharks is within $11 \%$ of the actual number of sharks. RMS errors in state estimation are higher when $10 \%$ of sharks are tagged. Although not included in the figure, the RMS error of estimated segment length stayed consistently within $12 \%$ of the actual segment length at steady state. When $10 \%$ of sharks are tagged, the line error exceeds the threshold of $0.1 \mathrm{~m} /$ shark. It should be noted that the Aggregation Particle Filter requires accurate estimates of $X_{i, t}$ and $X_{\text {line,t }}$, therefore an above-threshold line error results in a higher mean error in $n$ and $L$.

\subsection{Experiment Data Results}

The motivating video data set consisting of $n=112$ sharks and $L=30 \mathrm{~m}$ attraction line was also used to validate the state estimation process. Out of the 112 shark trajectories, the image processing algorithm was able to extract 92 trajectories. The position estimates $X_{t}$ from these trajectories 
Table 1: Particle Filter Parameters and APF Constants

\begin{tabular}{|l|l|l|l|l|l|l|l|l|l|l|l|l|l|}
\hline Parameter & $\sigma(m)$ & $\sigma_{L}(m)$ & $\sigma_{n}$ & $\sigma_{\text {line }}(m)$ & $\sigma_{\rho}(m)$ & $\sigma_{\psi}(m)$ & $p_{\text {crit }}(\%)$ & $K_{a t t}$ & $K_{\text {rep }}$ & $K_{\text {hab }}$ & $r_{\text {rep }}(m)$ & $r_{\text {neutral }}(m)$ & $r_{\text {att }}(m)$ \\
\hline Value & 5 & 10 & 10 & 20 & 1 & 1 & 90 & 1 & 57.5 & 205 & 2 & 5 \\
\hline
\end{tabular}

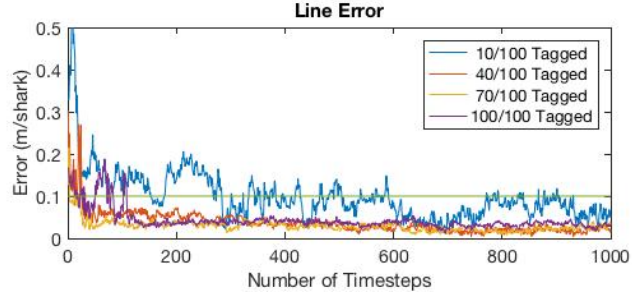

(a)

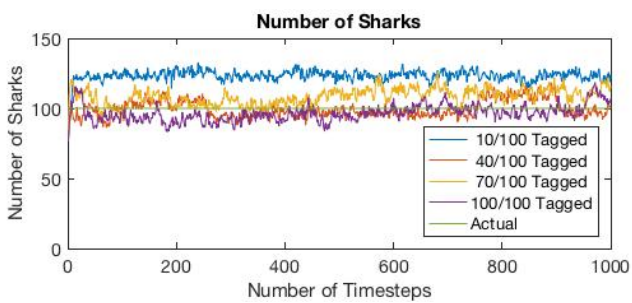

(b)

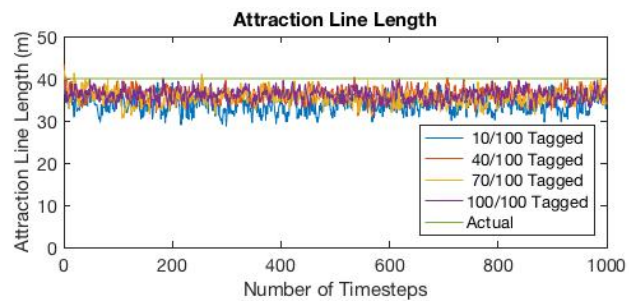

(c)

Figure 6: Simulation results with $n=100$ and $L=$ 40 for $m=10,40,70,100$. (a) shows line error, (b) shows estimated number of shark $n$, and (c) shows estimated attraction line length $L$.

were then processed using Algs. 1 and 2 to obtain estimates of $n$ and $L$ over time. For the purpose of these experiments, a random set of shark trajectories were said to be tagged. For example, to generate state estimation results for a case when only $10 \%$ of the sharks are tagged, a random set of $10 \%$ of the trajectories extracted from the video data set are used in conjunction with the algorithms.

The results are shown in Fig. 7 and the RMS state estimation errors are found in Table 3. The results show that, when $80 \%$ of sharks are tagged, the system is able to estimate the line, number of sharks $n$ and attraction line $L$. The line position estimation errors are consistently less than the threshold of $0.1 \mathrm{~m} /$ shark. The RMS error of estimated

Table 2: RMS error of simulation results

\begin{tabular}{|l|l|l|l|}
\hline$\epsilon_{m}$ (out of 100 Sharks) & $\epsilon_{\text {line }}(\mathrm{m} /$ shark $)$ & $\epsilon_{n}$ & $\epsilon_{L}(m)$ \\
\hline 100 & 0.03 & 6.48 & 4.17 \\
\hline 70 & 0.03 & 10.37 & 4.81 \\
\hline 40 & 0.03 & 6.07 & 4.03 \\
\hline 10 & 0.09 & 23.87 & 6.52 \\
\hline
\end{tabular}

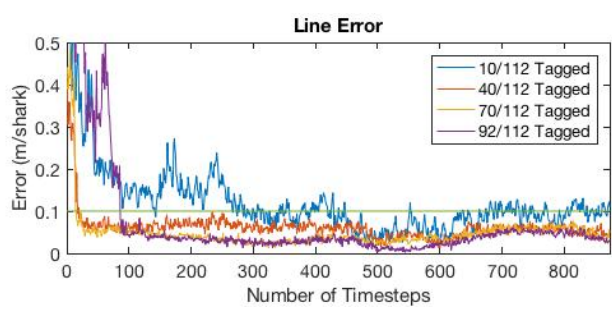

(a)

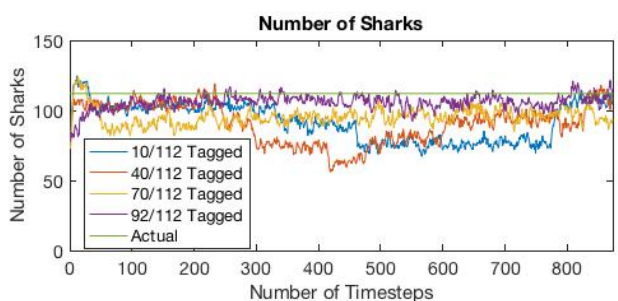

(b)

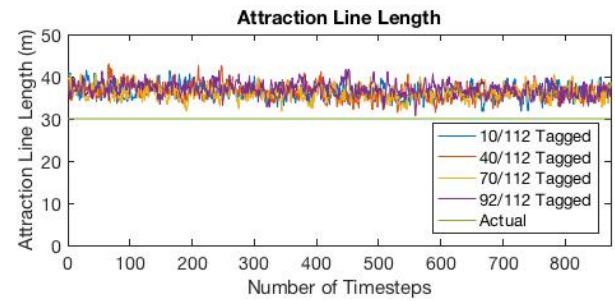

(c)

Figure 7: Experimental results for shark aggregation video data with $n=112$ and $L=30 m$ for $m=10,40,70,92$. (a) shows line error, (b) shows estimated number of shark $n$, and (c) shows estimated attraction line length $L$.

segment length is within $24 \%$ of the actual segment length at steady state. The estimated number of sharks is within $6 \%$ of the actual number of sharks. RMS errors in state estimation are higher when less than $80 \%$ of sharks are tagged.

\section{CONCLUSIONS AND FUTURE WORK}

The paper presents a method to track the position, orientation, and size of 2D animal aggregations. A model based on Markov Matrices built from historical data is presented as a viable model for representing aggregations. A hierarchical state estimator based on Particle Filtering is used to estimate the time-varying model parameters in real-time. The proposed method has been tested against simulation and has shown to estimate model parameters very well. In the motivating data set presented, sharks aggregate around a line of attraction. However, in another location where there is no constraining coastline, environment may include a point of attraction. For such cases, a similar approach can be used with a different model parameters. The ability to track an animal aggregation allows insight into the so- 
Table 3: RMS error of experimental results

\begin{tabular}{|l|l|l|l|}
\hline$\epsilon_{m}$ (out of 112 Sharks) & $\epsilon_{\text {line }}($ m/shark) & $\epsilon_{n}$ & $\epsilon_{L}(m)$ \\
\hline 92 & 0.03 & 6.63 & 7.16 \\
\hline 70 & 0.04 & 17.49 & 6.32 \\
\hline 40 & 0.05 & 27.01 & 6.42 \\
\hline 10 & 0.11 & 25.46 & 6.50 \\
\hline
\end{tabular}

cial behaviors and environmental dependence of the tagged fish. Long term changes to the population (ex. migration patterns, size changes) can be observed from a subset of individuals in the aggregation. The same process can be used for other stochastic group dynamics models (ex. Densitybased model) to estimate aggregation parameters. Future work for this project includes extending the process to track different animal aggregations, including barred sandbass, giant seabass and stingrays. Finally, we hope to deploy AUVs to conduct real-time, in situ tracking.

\section{Acknowledgment}

This material is based on work supported by the National Science Foundation under Grant No. 1423620 and by the USC Wrigley Institute. We also thank Benjamin Chasnov and Akhil Bagaria for their work on image processing.

\section{REFERENCES}

[1] D. Shinzaki, C. Gage, S. Tang, M. Moline, B. Wolfe, C. G. Lowe, and C. Clark. A multi-auv system for cooperative tracking and following of leopard sharks. In Robotics and Automation (ICRA), 2013 IEEE International Conference, pages 4153-4158, May 2013.

[2] Y. Lin, J. Hsiung, R. Piersall, C. White, C.G. Lowe, and C.M. Clark. A multi-autonomous underwater vehicle system for autonomous tracking of marine life. J. of Field Robotics, pages n/a-n/a, 2016.

[3] J. H. Tien, S. A. Levin, and D. I. Rubenstein. Dynamics of fish shoals: identifying key decision rules. Evolutionary Ecology Research, pages 555-565, 2004.

[4] Ichiro Aoki. A simulation study of the schooling mechanism in fish. Bulletin of the japanese society of scientific fisheries, 48(8):1081-1088, 1982.

[5] S. Gueron, S. A. Levin, and D. I. Rubenstein. The dynamics of herds: From individuals to aggregations. J. of Theo. Bio., 182(1):85 - 98, 1996.

[6] A. Okubo. Dynamical aspects of animal grouping: Swarms, schools, flocks, and herds. Advances in Biophysics, 22:1 - 94, 1986.

[7] C. W. Reynolds. Flocks, herds and schools: A distributed behavioral model. SIGGRAPH Comput. Graph., 21(4):25-34, August 1987.

[8] D. Grunbaum. Translating stochastic density-dependent individual behavior with sensory constraints to an eulerian model of animal swarming. J. of Mathematical Bio., 33:139-161, December 1994.

[9] C. Wissel A. Huth. The simulation of the movement of fish schools. J. of Theo. Bio., 156:365-385, June 1992.

[10] B. V. Hight and C. G. Lowe. Elevated body temperatures of adult female leopard sharks, triakis semifasciata, while aggregating in shallow nearshore embayments: Evidence for behavioral thermoregulation? J. of Experimental Marine Bio. and Ecology, 352(1):114 - 128, 2007.

[11] E.K. Kisafaludy M. A. Royer N.C. Wegner A. P. Nosal, A. Caillat. Aggregation behavior and seasonal philopatry in male and female leopard sharks triakis semifasciata along the open coast of southern california, usa. Ecol Prog Ser, 499:157-175, Mar. 2014.

[12] E.F. Morais, M.F.M. Campos, F.L.C. Padua, and R. L. Carceroni. Particle filter-based predictive tracking for robust fish counting. In XVIII SIBGRAPI'05, pages 367-374, Oct 2005.

[13] R.J. Petrell, X. Shi, R.K. Ward, A. Naiberg, and C.R. Savage. Determining fish size and swimming speed in cages and tanks using simple video techniques. Aquacultural Engineering, 16(1):63 - 84, 1997.

[14] B.P. Ruff, J.A. Marchant, and A.R. Frost. Fish sizing and monitoring using a stereo image analysis system applied to fish farming. Aquacultural Engineering, 14(2):155 - 173, 1995.

[15] Z. Qian and Y. Q. Chen X. Cheng. Automatically detect and track multiple fish swimming in shallow water with frequent occlusion. PLoS ONE, 9(9):1-12, 092014 .

[16] H. Cong, M. Sun, D. Zhou, and X. Zhao. Multi-target tracking of zebrafish based on particle filter. In 2016 35th Chinese Control Conference (CCC), pages 10308-10313, July 2016.

[17] M. Espinoza, T. J. Farrugia, D. M. Webber, F. Smith, and C. G. Lowe. Testing a new acoustic telemetry technique to quantify long-term, fine-scale movements of aquatic animals. Fisheries Research, 108(2 - 3):364 - 371, 2011.

[18] S. Clements, D. Jepsen, M. Karnowski, and C. B. Schreck. Optimization of an acoustic telemetry array for detecting transmitter-implanted fish. N. American J. of Fisheries Management, 25(2):429-436, 2005.

[19] J. D. Peterson K. N. Holland, C. G. Lowe and A. Gill. Tracking coastal sharks with small boats: Hammerhead shark pups as a case study. Marine and Freshwater Research, pages 61-66, 1992.

[20] T. M. Grothues, J. Dobarro, and J. Eiler. Collecting, interpreting, and merging fish telemetry data from an auv: Remote sensing from an already remote platform. In 2010 IEEE/OES Autonomous Underwater Vehicles, pages 1-9, Sept 2010.

[21] M. J. Oliver, M. W. Breece, D. A. Fox, D. E. Haulsee, J. T. Kohut, J. Manderson, and T. Savoy. Shrinking the haystack: Using an auv in an integrated ocean observatory to map atlantic sturgeon in the coastal ocean. Fisheries, 38(5):210-216, 2013.

[22] H. Bayram, J. V. Hook, and V. Isler. Gathering bearing data for target localization. IEEE Robotics and Automation Letters, 1(1):369-374, Jan 2016.

[23] G.E. Packard, A. Kukulya, T. Austin, M. Dennett, R. Littlefield, G. Packard, M. Purcell, R. Stokey, and G. Skomal. Continuous autonomous tracking and imaging of white sharks and basking sharks using a remus-100 auv. In 2013 OCEANS, pages 1-5, Sept 2013.

[24] T. Hodge R. Robeva. Mathematical Concepts and Methods in Modern Bio.: Using Modern Discrete Models. Academic Press, 2013. 